\title{
Upper limb kinematics in stroke and healthy controls using target-to-target task in virtual reality
}

Netha Hussain, Margit Alt Murphy, Katharina S Sunnerhagen Institute of Neuroscience and Physiology University of Gothenburg, Sweden
Contact information

Netha Hussain

netha.hussain@gu.se

\section{Background}

Kinematic assessment involving the use of a haptic device in a virtual reality environment is suitable for measuring the upper limb impairment after stroke.

\section{Aim}

To identify the end-point kinematic variables obtained using the target-to-target pointing task that discriminate among individuals with mild and moderate upper limb impairment after stroke and healthy controls.

\section{Methods}

Participants: 67 individuals with moderate (32-57 points) to mild stroke (58-65 points) impairment based on Fugl-Meyer Assessment of Upper Extremity and 43 healthy controls.

The individuals with stroke were extracted from Stroke Arm Longitudinal study at the University of Gothenburg (SALGOT).

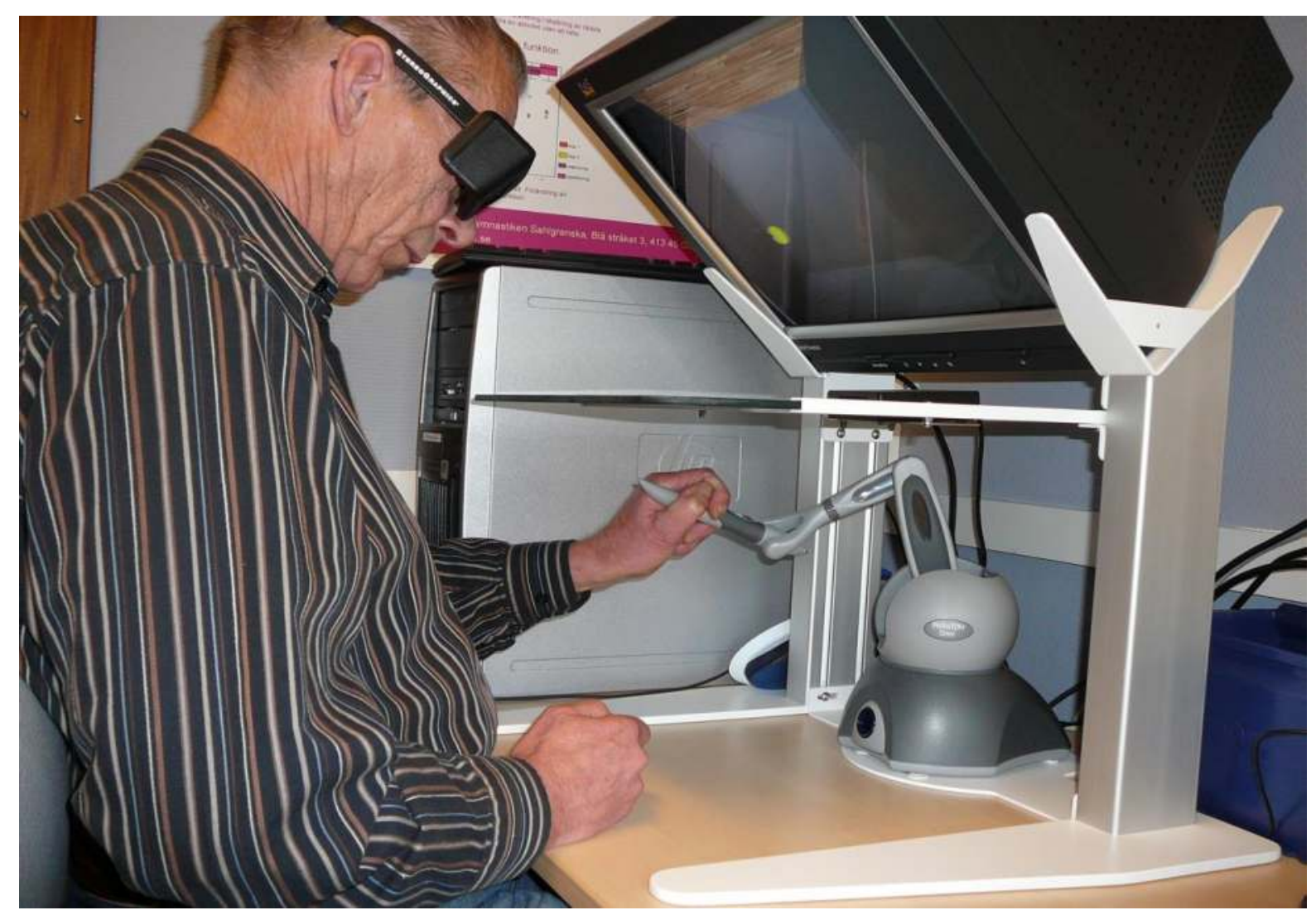

A participant is performing the target-to-target pointing task, where circular targets appear one after the other and disappear when pointed at by the haptic stylus in a three dimensional virtual environment.

\section{Results}

The movement time, mean velocity and number of velocity peaks were discriminative between individuals with mild and moderate stroke impairment. Additionally, the number of velocity peaks was also discriminative for individuals with stroke and healthy controls.

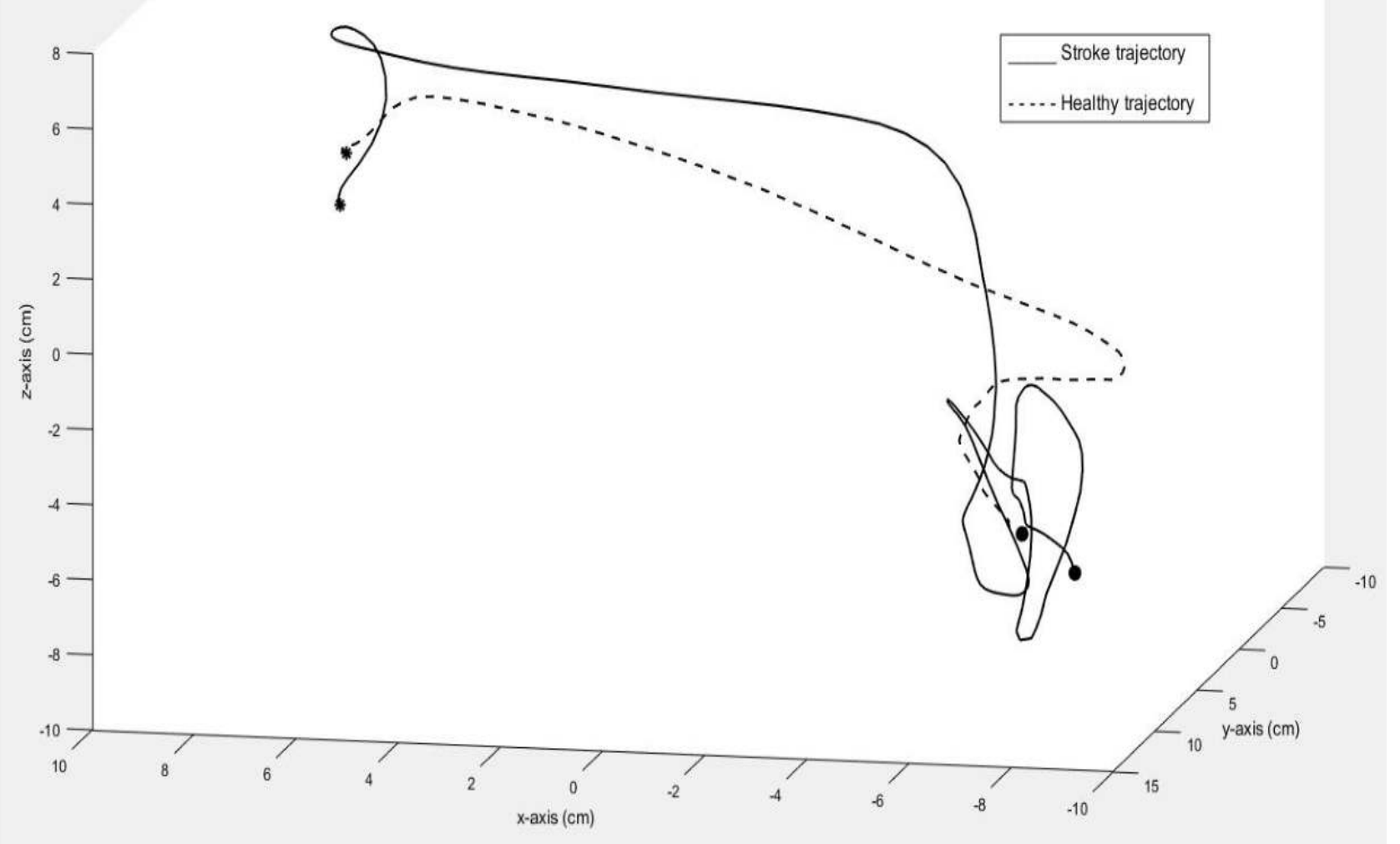

Trajectory of one individual with moderate stroke impairment and one healthy control. In the stroke individual, spider-web like convolusions are seen towards the end of the movement.

\section{Conclusion}

The target-to-target task can discriminate among mild and moderate motor sensorimotor impairment of the upper limb in individuals after stroke and healthy controls.

The virtual reality based targetto-target task is useful for the assessment of arm motor impairment following stroke. 